\title{
Continuous cold exposure induces an anti-inflammatory response in mesenteric adipose tissue associated with catecholamine production and thermogenin expression in rats
}

\author{
${ }^{1}$ Vargovic P, ${ }^{2}$ Manz G, ${ }^{1}$ Kvetnansky R \\ ${ }^{1}$ Institute of Experimental Endocrinology, Biomedical Research Center, Slovak Academy of Sciences, Bratislava, Slovakia; \\ ${ }^{2}$ LDN Labor Diagnostica Nord, D-48531, Nordhorn, Germany \\ E-mail: peter.vargovic@savba.sk
}

Objective. Continuous exposure to cold leads to an activation of adaptive thermogenesis in the brown adipose tissue and induction of brown/beige cell phenotype in the white adipose tissue. Thermogenic response is associated with alternatively activated macrophages producing catecholamines, which subsequently activate the uncoupling protein 1 (UCP-1). The aim of this work was to elucidate the effect of cold exposure on catecholamine and immune responses associated with adipocyte browning in the mesenteric adipose tissue (mWAT) of rat.

Methods. The rats were exposed to continuous cold $\left(4^{\circ} \mathrm{C}\right)$ for 1 or 7 days. Catecholamines production and gene expressions of inflammatory and other factors, related to adipocyte "browning", were analyzed in the homogenized mWAT samples using 2-CAT ELISA kits.

Results. Cold exposure induced a sympathetic response in the mWAT, evidenced by the tyrosine hydroxylase $(\mathrm{TH})$ protein level rise. Induction of non-sympathetical catecholamine production was observed 7 days after cold exposure by elevated TH and phenylethanolamine-N-methyltransferase (PNMT) expression, leading to an increased epinephrine levels. Cold exposure for 7 days stimulated the infiltration of macrophages, evaluated by F4/80 and CD68 expressions, and expression of anti-inflammatory mediators, while pro-inflammatory cytokines were inhibited. Anti-inflammatory response, accompanied by de novo catecholamine production and up-regulation of $\beta 3$-adrenergic receptors, led to the stimulation of UCP-1 and PGC1 1 expression, suggesting a cold-induced "browning" of the mWAT, mediated by alternatively activated macrophages.

Conclusions. The present data indicate that prolonged cold exposure may induce anti-inflammatory response in mWAT associated with induction of UCP-1 expression. Although functional thermogenesis in the mWAT is most likely redundant, a highly efficient dissipation of energy by $\mathrm{UCP} 1$ may affect the energy homeostasis in this visceral fat.

Key words: adipose tissue, catecholamines, cold exposure, thermogenesis, inflammatory mediators

Adipose tissue belongs to the active visceral systems efficiently contributing to not only the metabolic but also immuno-endocrine processes and regulations. Visceral adipose tissue exhibits high immuno-endocrine activity due to production of cytokines and adipokines by adipocytes and activity of infiltrated immune cells (e.g. tissue macrophages, T-cells, B-cells, eosinophils, type 2 innate lymphoid cells, etc.). Obesity, if accompanied by a visceral adiposity, is associated with the visceral fat proinflammatory activity that is strongly related to the metabolic dysfunction and systemic inflammation

Corresponding author: Peter Vargovic, PhD., Institute of Experimental Endocrinology, Biomedical Research Center, Slovak Academy of Sciences, Dubravska cesta 9, 84505 Bratislava 4, Slovakia, phone: +421-2-32295279, e-mail: peter.vargovic@gmail.com. 
(Miyazaki et al. 2002; Sam et al. 2009). Epidemiological studies have indicated that mainly abundance of visceral fat is related to the development of the type 2 diabetes mellitus and cardiovascular disease (Nicklas et al. 2004; Kanhai et al. 2012). One of the visceral fats is represented by the mesenteric white adipose tissue (mWAT), which exhibits a significant immuno-endocrine activity in the diabetes, metabolic syndrome, and inflammatory bowel disease (Yang et al. 2008; Bertin et al. 2010; Batra et al. 2012).

Recent obesity research has been focused on the thermogenic ability of the brown adipose tissue (BAT) that is able to produce heat through uncoupling fatty acid oxidation from adenosine-5'-triphosphate production by UCP-1. The major physiological regulator of BAT thermogenesis is $\beta 3$-adrenergic stimulation by norepinephrine (NE) released from the sympathetic nerves (Young et al. 1984). The browning process can be mimicked by chronic treatment with $\beta 3$-adrenergic receptor agonists (Cousin et al. 1992). Some of the mouse and human white adipose tissues contain cells that can express functionally uncoupling protein UCP-1. These cells are called as brownin-white (brite) cells, beige cells or adaptive and recruitable brown adipocytes (Seale et al. 2008; Enerback 2009). The beige adipocytes show a multilocular morphology as well as other brown-characteristic upon prolonged stimulation by cold or pathways that may elevate the intracellular cyclic AMP. Exposure of adult animals to an environmental cold switches on the catabolic programs in the subcutaneous white adipose tissue (scWAT) in mice, which support the thermogenesis and growth of thermogenic beige fat via activating the adrenergic signaling pathways ( $\mathrm{Wu}$ et al. 2012; Harms and Seale 2013).

Recently, several studies have pointed out the immune cells importance in the regulation of beige fat adipogenesis and thermogenesis in response to cold exposure. The mechanisms include secretion of IL-4 by eosinophils, which stimulate an alternative activation of macrophages and their catecholamine (CA) production (Nguyen et al. 2011; Qiu et al. 2014), and the activation of type 2 innate lymphoid cells by IL-33, leading to proliferation of the bipotential adipocyte precursors and their subsequent commitment to the beige fat lineage (Lee et al. 2015).

CA production in the adipose tissue cells, particularly in the alternatively activated M2 macrophages, appears as an essential mechanism of adipose tissuerelated thermogenesis that maintains the adrenergic tone additionally to the activity of the sympathetic system. We have identified CA biosynthetic enzymes expression in the brown and white adipose tissues and described the stimulation of CA production in the cell fractions of the mesenteric adipose tissue in response to immobilization or cold stress (Vargovic et al. 2011; Kvetnansky et al. 2012; Vargovic et al. 2013; Vargovic et al. 2016). Since only the mWAT showed stress-inducible cell-specific CA production, from all the investigated adipose tissues (brown, epididymal, retroperitoneal, subcutaneous), we suppose that the mWAT may represent another active site of the beige adipogenesis, similarly as it has been described in the case of the scWAT.

The aim of this study was to reveal the gene expression features of the beige fat in the mWAT and to find out whether 1 or 7 days cold exposure may affect de novo CA production and immune responses associated with thermogenesis.

\section{Material and Methods}

Animals. Male Sprague-Dawley rats, 4 months old and weighing 300-350 g (Charles River, Suzfeld, Germany), were used in the experiments. Animals were housed 3-4 per cage in a controlled environment $\left(23 \pm 1^{\circ} \mathrm{C}, 12 \mathrm{~h}\right.$ light-dark cycle, lights on at $\left.6 \mathrm{AM}\right)$. All the experimental procedures and animal treatments used have been approved by the Ethics Committee of the Institute of Experimental Endocrinology (Slovak Academy of Sciences, Bratislava, Slovakia) under the No. RO-2804/07-221/3.

Cold exposure. Animals were housed two per cage in a controlled environment $\left(23 \pm 1^{\circ} \mathrm{C}, 12 \mathrm{~h}\right.$ light-dark cycle, lights on at $6 \mathrm{AM}$ ) for 7 days before starting the experiments. Cold-exposed rats were transferred into a cold room $\left(4 \pm 1^{\circ} \mathrm{C}, 12 \mathrm{~h}\right.$ light-dark cycle, lights on at $6 \mathrm{AM}$ ) for 1 day or continual exposure for 7 days. These experimental groups were sacrificed by decapitation directly in the cold room at the same day as the controls housed at $23^{\circ} \mathrm{C}$.

Catecholamine determination. Catecholamines were analyzed in the mWAT samples, which were homogenized in $0.01 \mathrm{M} \mathrm{HCl}$. Homogenates supernatants were used for the catecholamine measurements using 2-CAT ELISA kits (Labor Diagnostica Nord, Nordhorn, Germany) as described previously (Vargovic et al. 2011). The values were normalized to proteins in homogenate, determined by bicinchoninic acid (BCA) Protein Assay (Thermo Scientific, Rockford, IL).

RNA isolation and relative quantification of mRNA levels by real time RT-PCR. Total RNA from the mWAT samples was isolated by TRI Reagent (MRC Ltd., Cincinnati, OH, USA), as described previously (Vargovic et al. 2011). Reverse transcrip- 
tion and real time PCR were also performed, as described previously (Vargovic et al. 2013). For all PCR amplifications, aliquots containing $20 \mathrm{ng}$ of total RNA were used. TH and PNMT were amplified using specific TaqMan probes (Applied Biosystems) (Rn00562500_ml for TH, Rn01495588_m1 for PNMT) and Maxima Probe/ROX qPCR Master Mix in $10 \mu \mathrm{l}$ of total volume, according to the manufacturer's protocol. All the other transcripts were amplified using SYBR Green Mastermix (ThermoFischer Scientific) with specific primers (5 pmol) in $10 \mu \mathrm{l}$ of final volume. The primer sequences are displayed in Table. 1. Data were analyzed with SDS software version 2.3 (Applied Biosystems) and inspected to determine artifacts (loading errors, threshold errors, etc). Baseline levels for each gene were computed automatically. Count numbers (Ct values) were exported to an Excel spreadsheet and analyzed according to the $\Delta \Delta \mathrm{CT}$ method described by Livak and Schmittgen (2001). Expressions of analyzed transcripts were normalized to average values of three internal controls, i.e. tatabinding protein (TBP), glyceraldehyde-3-phosphate dehydrogenase (GAPDH) and rps29.

Western blot analysis. The mWAT samples (100$200 \mathrm{mg}$ ) were homogenized in $2 \mathrm{ml}$ of $20 \mathrm{mM}$ Tris pH 7.0, 5 mM PMSF (Pefabloc SC + Compete, Roche Diagnostic), incubated on ice for $1 \mathrm{~h}$ and centrifuged for $15 \mathrm{~min}\left(16100 \times \mathrm{g}, 4^{\circ} \mathrm{C}\right)$ in order to extract the cytosolic fraction. Western blot was performed, as described previously (Laukova et al. 2010). Glyceraldehyde-3-phosphate dehydrogenase (GAPDH) was used as an internal and loading control, since it was not affected by the experimental conditions. The following primary antibodies were used: monoclonal anti-TH (1:3000; MAB5280; Chemicon International, Temecula, CA) and monoclonal anti-GAPDH (1:5000, MAB374, Chemicon International, Temecula, CA). Membranes were incubated with anti-mouse horseradish peroxidase-linked secondary antibodies diluted 1:5000 for $1 \mathrm{~h}$ at room temperature. The signals were detected using Femto Supersignal reagent (Pierce, Rockford, Illinois) and visualized by exposure to Amersham Hyperfilm (Amersham Biosciences). Optical density of individual bands (o.d. $\left./ \mathrm{mm}^{2}\right)$ was quantified by PC BASE 2.08e software (Raytest, Inc.) and normalized to GAPDH.

Statistical analysis. Statistical differences between two experimental groups were determined by T-test, while statistical differences among more than two groups were determined by one-way and two way analysis of variance (ANOVA), followed by Bonferonni post hoc test [SigmaStat, version 3.1, Systat Software, Inc., USA]. Results are presented as mean \pm
S.E.M. and each value represents $6-8$ rats. Values of ${ }^{*} \mathrm{p}<0.05,{ }^{*} \mathrm{p}<0.01$ defined the statistical significance vs. control group.

\section{Results}

At first, we examined the catecholaminergic system response to cold in the mWAT (Figure 1). Cold exposure did not affect the NE concentration but elevated the epinephrine (EPI) level after 7 days of cold $(\mathrm{p}<0.01$, Figure 1). TH, which is the first and rate-limiting enzyme involved in catecholamine biosynthesis, showed increased protein level in response to 1 day $(\mathrm{p}<0.05)$ or 7 days $(\mathrm{p}<0.01)$ cold exposure. In order to assess de novo synthesis of CAs in the mWAT, we analyzed TH and PNMT gene expression, which may reflect non-sympathetic sources of CA production in macrophages and adipocytes, as it has been described previously (Nguyen et al. 2011; Vargovic et al. 2011; Vargovic et al. 2013). Real time RT-PCR analysis revealed that 7 days cold induced a rise of TH $(\mathrm{p}<0.01)$ and PNMT $(\mathrm{p}<0.05)$ expression.

Next, we examined the expression of $\beta 3$ adrenergic receptors ( $\beta 3$-ARs), which activation is essential for release of free fatty acids to fuel uncoupled respiration and stimulation of the expression of thermogenic genes, such like PPAR-c coactivator $1 \alpha$ (PGCla) and uncoupling protein 1 (UCP1) (Cannon and Nedergaard 2004). One or 7 days cold exposure induced rise in $\beta 3$-ARs mRNA (both $\mathrm{p}<0.05$ ). In order to assess whether cold exposure may stimulate an alternative activation of the mWAT tissue macrophages by interleukin 4 (IL-4) leading to activation of thermogenic gene expression as described by Nguyen et al. (2011), we determined the expression of macrophages markers F4/80 and CD68 (Khazen et al. 2005) and expression of the pro-inflammatory and anti-inflammatory factors in the mWAT (Figure 2). One or 7 days cold exposure stimulated the infiltration of macrophages demonstrated by elevated of F4/80 (both $\mathrm{p}<0.05$ ) and CD68 (both $\mathrm{p}<0.05$ ) expression. Furthermore, 7 days lasting cold exposure induced expression of the antiinflammatory markers, i.e. arginasel $(p<0.05)$, interleukin 4 (IL-4, p<0.05), and interleukin 10 (IL-10, $\mathrm{p}<0.05)$, while it reduced the pro-inflammatory cytokines, including interleukin 6 (IL-6, p<0.05), interleukin $1 \beta$ (IL-1 $\beta, \mathrm{p}<0.05$ ), and interleukin 17 (IL-17, $\mathrm{p}<0.05$ ) in the mWAT (Figure 2).

To find out whether cold-stimulated activity of catecholamine system, accompanied by alternative activation of macrophages, is also associated with the main features of the thermogenic response, we examined the expression of the UCP-1 and PGC-1a 
Figure 1. Effect of cold exposure on sympathetic or non-sympathetic catecholamine production in mesenteric adipose tissue. Control rats (C) or rats exposed to continuous 1- or 7 -days cold. (a) Norepinephrine (NE) and epinephrine (EPI) concentrations in mWAT. (b) Western blot of tyrosine hydroxylase $(\mathrm{TH})$ in protein homogenates $(10 \mu \mathrm{g}$ of soluble cytosolic fraction) of mWAT. Loading control: glyceraldehyde-3-phosphate dehydrogenase (GAPDH). (c) Gene expression of TH and PNMT reflecting non-sympathetical catecholamine production in mWAT. (d) Gene expression of $\beta 3$-subtype of adrenergic receptors. Each column is displayed as mean \pm S.E.M. and represents an average of 6-8 animals. Values of ${ }^{*} \mathrm{p}<0.05$ or ${ }^{* *} \mathrm{p}<0.01$ defined the statistical significance vs. control group.

\section{a}

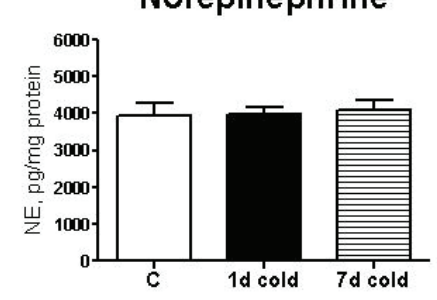

b
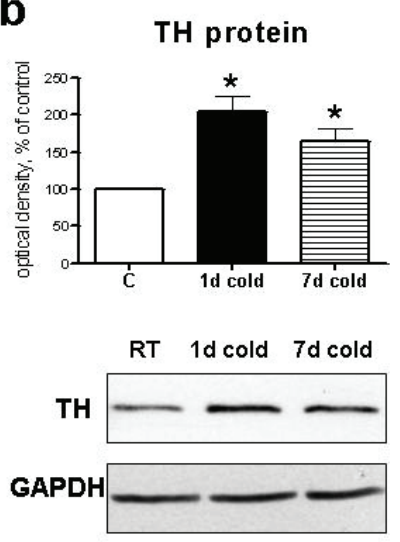

Epinephrine

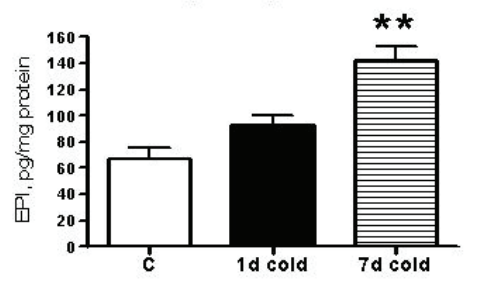

C

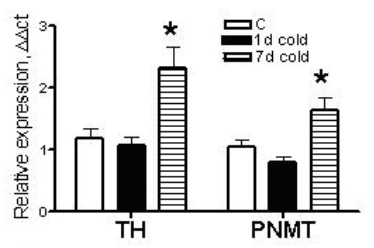

d

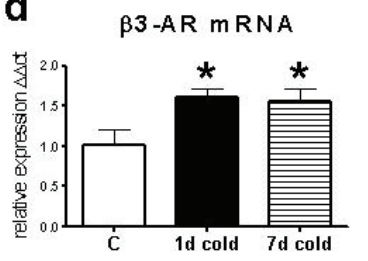

thermogenic genes. Although 1 day cold exposure did not change their levels, 7 days lasting cold significantly elevated both the UCP1 $(\mathrm{p}<0.01)$ and PGC-1a $(\mathrm{p}<0.01)$ mRNA levels. Furthermore, gene expressions of IL-4 and UCP-1 showed significant positive correlation $(\mathrm{R}=0.74, \mathrm{p}<0.001, \mathrm{~N}=17)$.

\section{Discussion}

The ability of the brown or beige fat to suppress the obesity through increased energy expenditure has induced an interest explosion in the adipose tissueassociated adaptive thermogenesis studies. Most of the studies have been focused on the mechanisms of thermogenesis activation in BAT or brown-like cells present in the scWAT, which ability to thermogenesis represent an important mechanism in maintaining the body temperature in the adult animals. Although the ability of adaptive thermogenesis in visceral fat appears as a redundant, potential uncoupling activity of UCP1 could significantly participate in the catabolic activity of the fat. Importantly, recent studies have indicated that "beige" adipocyte activity may affect the systemic metabolism and contribute significantly to the whole body insulin sensitivity (Qiu et al. 2014, Cohen et al. 2014). The UCP-mediated catabolic activity appears to be a most useful in the fight against the abdominal visceral obesity, which is associated with the insulin resistance and metabolic syndrome. From all the visceral adipose tissues, the mWAT mass, when increased, exhibits inflammation features related to both the insulin resistance and metabolic syndrome in contrast to other visceral fat depots (Kranendonk et al. 2015). The mWAT, particularly in visceral adiposity, shows high immune and endocrine activities by adipocytes themselves as well as due to local activity of a large number of infiltrated immune cells, particularly pro-inflammatory M1 macrophages. Since immune activity, particularly alternative activation of macrophages by IL-4, plays a significant role in the activation of UCP1-mediated adaptive thermogenesis in BAT and scWAT. There is a high probability to find analogous processes in the interactions between immune and fat cells in the mWAT. Previously we have described the highest NE and EPI concentrations in the mWAT, in comparison with other visceral or subcutaneous WAT depots. The quick ability of stress-induced production of sympathetic or non-sympathetic CAs is essential in the UCP1 activation (Kvetnansky et al. 2012; Vargovic et al. 2013).

In the present study, we showed that cold exposure appears to be not only a significant inductor of the sympathetic activity in the mWAT (as evidenced by $\mathrm{TH}$ protein level rise), but also in the stimulation of 
a

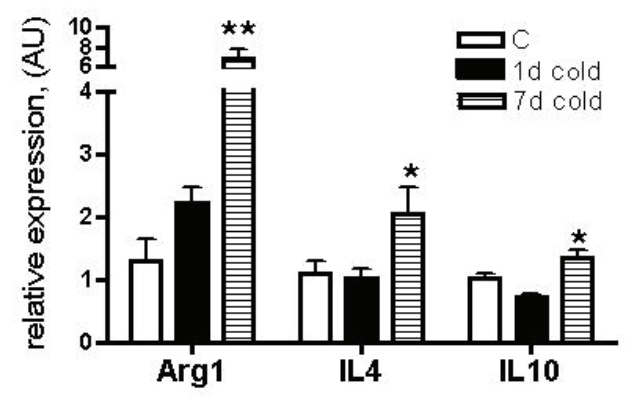

b

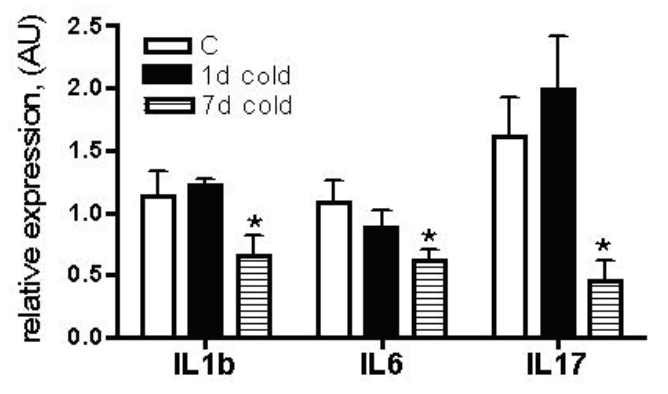

e

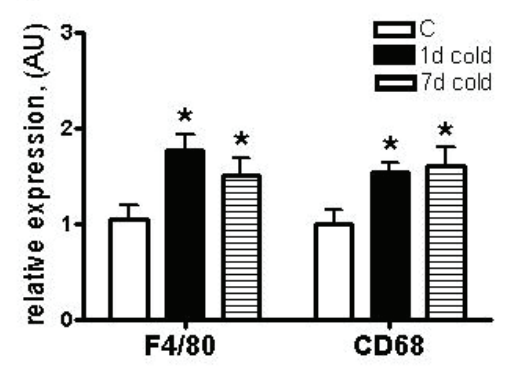

d

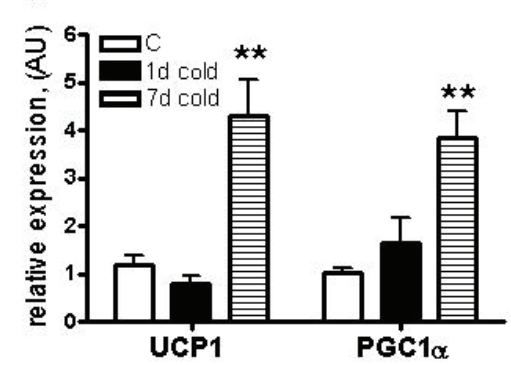

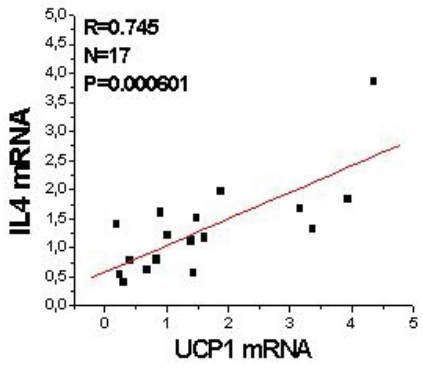

Figure 2. Cold induced immune response and thermogenesis-associated expression changes in mWAT. Control rats (C) or rats exposed to 1- or 7-days continuous cold. (a) Gene expression of anti-inflammatory factors: Arginase 1 (Arg1), interleukin 4 (IL4), interleukin 10 (IL10). (b) Gene expression of pro-inflammatory factors: interleukin $1 \mathrm{~b}$ (IL1b), interleukin 6 (IL6), interleukin 17 (IL17). (c) Gene expression of macrophages markers F4/80 and CD68. (d) Gene expression of thermogenic genes: uncoupling protein (UCP1), PGC1a. (e) Correlation between UCP1 and IL4 gene expressions. Values are normalized to internal control (18 S rRNA) and presented as fold change relative to control values evaluated by $\Delta \Delta \mathrm{Ct}$ method. Each column is displayed as mean \pm S.E.M. and represents an average of 6-8 animals. Values of ${ }^{\star} p<0.05$ or ${ }^{* *} p<0.01$ defined the statistical significance vs. control group.

TH and PNMT expression suggesting a non-sympathetic cold-inducible CA production (Figure 1). This is also supported by observation of cold-elevated EPI tissue level. NE that is produced mostly by the sympathetic nerves remained unchanged probably due to the quick physiological mechanisms of NE supplies maintenance consisting of NE turnover regulation. Due to a very high concentration of sympathetic NE stores within the mWAT compared to NE produced by adipose tissue cells such as macrophages or adipocytes (Vargovic et al. 2011), it is probably impossible to distinguish the changes in non-sympathetic NE unless the mWAT is separated into the cell fractions (Vargovic et al. 2011). However, in our previous study we have described cold-induced elevations of NE and EPI levels in adipocytes and stromal/vascular fractions, obtained from the mWAT by collagenase digestion (Kvetnansky et al. 2012). Furthermore, we have identified cold-induced stimulation of catechol- amine synthesizing enzymes, TH and PNMT, in the stromal/vascular fraction but not in the adipocytes. Considering the previous data in stromal/vascular cells, which are rich in macrophages, the primary source of the cold-induced CA production should be in the mWAT. Therefore, in the present study, we did fractionalize the mWAT into adipocyte and stromal/ vascular fractions. We focused on CA production in the mWAT expecting the macrophages as the main source of non-sympathetic CAs in the mWAT. This is in accordance with Nguyen at al. (2011) who have demonstrated that cold exposure may stimulate the alternative activation of the tissue macrophages in the BAT and WAT, which in turn produces CA to sustain adaptive thermogenesis via UCP-1 activity. The key link in this process is mediated by eosinophilsderived IL-4, which induces TH expression in macrophages (Qiu et al., 2014). We observed a rise in the expression of IL-4 and other anti-inflammatory factors 
(arginase 1, interleukin IL-10) induced by 7 days cold exposure suggesting analogous processes running in the mWAT (Figure 2). Simultaneous down-regulation of pro-inflammatory factors, including IL-1 $\beta$, IL-6, and IL-17, supports a paradigm of the cold-induced anti-inflammatory programming in the mWAT, too. These changes might most likely result in the stimulation of UCP-1 expression (Figure 2). Similarly, we also found a cold-induced increase in the expression of PGC-1a, which is the central regulator in the expression of UCP-1 and other thermogenic genes in both the brown and beige fat cells (Lin et al. 2004; Kleiner et al. 2012).

In addition, we also identified a cold-induced rise in macrophage markers F4/80 and CD68 suggesting cold-stimulated infiltration of macrophages into the mWAT. Elevated expression of both anti-inflammatory factors, while dropped that of pro-inflammatory cytokines, suggest a dominant presence of the M2 (anti-inflammatory) macrophages. In the lean mice and humans, the adipose-tissue macrophages (ATM) are characterized by high arginase 1 and IL-10 expression that is specific for alternatively polarized, or M2 anti-inflammatory phenotype (Lumeng et al. 2007). Obesity induces an accumulation of classically polarized or M1 inflammatory, ATMs, leading to a pro-inflammatory state in adipose tissues and insulin resistance (Weisberg et al. 2003; Olefsky and Glass 2010). The regulation of M1 vs. M2 macrophage polarization, especially in vivo, is a balancing act that is still not fully understood. Recent studies have suggested that the M1-M2 switch in adipose tissues is caused by differential recruitment of various monocyte subtypes (Lumeng et al. 2008). Since we have previously found correlation between $\mathrm{F} 4 / 80$ and $\mathrm{TH}$ expression in the mWAT, rising number of M2 macrophages may participate in CA production (Vargovic et al. 2016). Elevated monocyte infiltration in WAT is observed especially in obesity but with dominant M1 phenotype associated with inflammation, resistin expression, and pathological consequences related to insulin resistance. Cold exposure-driven macrophage infiltration may also be somehow connected to an increased sympathetic activity leading to catabolism in the adipose tissue. Treatment with a $\beta 3$-adrenergic agonist triggers the death of numerous unilocular white adipocytes, followed by clearance of the dead fat cells (efferocytosis) by resident macrophages that assume an M2 phenotype and a subsequent brown/ beige adipogenesis in gonadal white adipose tissue (Lee et al. 2012; 2013). Liu et al. (2015) have shown that receptor Interacting Protein 140 (RIP140) may play a key role in regulating the balance of M1/M2 macrophages in the white adipose tissue. Lowering the level of RIP140 in macrophages reduces M1 macrophages, while it expands anti-inflammatory M2 macrophages, triggering WAT "browning", fat burning, and restoration of insulin sensitivity. Therefore, activation of the anti-inflammatory pathways as well as UCP-mediated "burning" of fat suggests a potential treatment of metabolic syndrome. Potentially, effective therapeutic approach would consist of reprogramming steps of the mWAT white phenotype of adipocytes to the beige ones. Effect of UCP-mediated dissipation of energy could be an efficient way to decrease the WAT mass, especially in the visceral fat depots, which are from all the fat depots mostly associated with the health problems risks and diseases, such as insulin resistance, diabetes, and cardiovascular disease (Kranendonk et al. 2015).

In summary, we are showing that 7 days lasting exposure to cold induces a specific immune response in the mWAT, including macrophages infiltration, induction of their anti-inflammatory phenotype, and catecholamine production, resulting to gene expression changes associated with adipocyte browning and thermogenic response. The data suggest that cold exposure-induced mechanisms of adipocyte browning in mWAT may contribute to novel therapeutic approaches of the metabolic syndrome treatment or other diseases related to the mWAT adiposity. However, the physiological significance and detailed mechanisms of occurred paradigm, should be clarified in further studies.

\section{Acknowledgement}

This research was supported by Slovak Research and Development Agency (No. APVV-0088-10); and VEGA Grant (2/0067/14).

\section{References}

Batra A, Heimesaat MM, Bereswill S, Fischer A, Glauben R, Kunkel D, Scheffold A, Erben U, Kuhl A, Loddenkemper C, Lehr HA, Schumann M, Schulzke JD, Zeitz M, Siegmund B. Mesenteric fat - control site for bacterial translocation in colitis? Mucosal Immunol 5, 580-591, 2012Bertin B, Desreumaux P, Dubuquoy L. Obesity, visceral fat and Crohn's disease. Curr Opin Clin Nutr Metab Care 13, 574-580, 2010. 
Cannon B, Nedergaard J. Brown adipose tissue: function and physiological significance. Physiol Rev 84, 277-359, 2004.

Cohen P, Levy JD, Zhang Y, Frontini A, Kolodin DP, Svensson KJ. Ablation of PRDM16 and beige adipose causes metabolic dysfunction and a subcutaneous to visceral fat switch. Cell 156, 304-316, 2014.

Cousin B, Cinti S, Morroni M, Raimbault S, Ricquier D, Penicaud L, Casteilla L. Occurrence of brown adipocytes in rat white adipose tissue: molecular and morphological characterization. J Cell Sci 103 (Pt 4), 931-942, 1992.

Enerback S. The origins of brown adipose tissue. N Engl J Med 360, 2021-2023, 2009.

Harms M, Seale P. Brown and beige fat: development, function and therapeutic potential. Nat Med 19, 1252-1263, 2013.

Kanhai DA, Kappelle LJ, van der Graaf Y, Uiterwaal CS, Visseren FL. SMART Study Group, The risk of general and abdominal adiposity in the occurrence of new vascular events and mortality in patients with various manifestations of vascular disease, Int J Obes 36, 695-702, 2012.

Khazen W, M'Bika JP, Tomkiewicz C, Benelli C, Chany C, Achour A, Forest C. Expression of macrophage-selective markers in human and rodent adipocytes. FEBS Lett 579, 5631-5634, 2005.

Kleiner S, Mepani RJ, Laznik D, Ye L, Jurczak MJ, Jornayvaz FR. Development of insulin resistance in mice lacking PGC-1alpha in adipose tissues. Proc Natl Acad Sci USA 109, 9635-9640, 2012.

Kranendonk ME, van Herwaarden JA, Stupkova T, de Jager W, Vink A, Moll FL, Kalkhoven E, Visseren FL.Inflammatory characteristics of distinct abdominal adipose tissue depots relate differently to metabolic risk factors for cardiovascular disease: distinct fat depots and vascular risk factors. Atherosclerosis 239, 419-427, 2015.

Kvetnansky R, Ukropec J, Laukova M, Manz B, Pacak K, Vargovic P. Stress stimulates production of catecholamines in rat adipocytes. Cell Mol Neurobiol 32, 801-813, 2012.

Laukova M, Vargovic P, Krizanova O, Kvetnansky R. Repeated stress down-regulates $\beta(2)$ - and a (2C)-adrenergic receptors and up-regulates gene expression of IL-6 in the rat spleen. Cell Mol Neurobiol 30, 1077-1087, 2010.

Lee YH, Petkova Anelia P, Mottillo Emilio P, Granneman JG. In vivo identification of bipotential adipocyte progenitors recruited by beta 3-adrenoceptor activation and high-fat feeding. Cell Metab 15, 480-491, 2012.

Lee YH, Petkova Anelia P, Granneman JG. Identification of an adipogenic niche for adipose tissue remodeling and restoration. Cell Metab 18, 355-367, 2013.

Lee MW, Odegaard JI, Mukundan L, Qiu Y, Molofsky AB, Nussbaum JC, Yun K, Locksley RM, Chawla A. Activated type 2 innate lymphoid cells regulate beige fat biogenesis. Cell 160, 74-87, 2015.

Lin J, Wu PH, Tarr PT, Lindenberg KS, St-Pierre J, Zhang CY. Defects in adaptive energy metabolism with CNSlinked hyperactivity in PGC-1alpha null mice. Cell 119, 121-135, 2004.

Liu PS, Lin YW, Burton FH, Wei LN. M1-M2 balancing act in white adipose tissue browning - a new role for RIP140. Adipocyte 4, 146-148, 2015.

Livak KJ, Schmittgen TD. Analysis of relative gene expression data using real-time quantitative PCR and the 2- $\Delta \Delta \mathrm{CT}$ method. Methods 25, 402-408, 2001.

Lumeng CN, Bodzin JL, Saltiel AR. Obesity induces a phenotypic switch in adipose tissue macrophage polarization. J Clin Invest 117, 175-184, 2007.

Lumeng CN, DelProposto JB, Westcott DJ, Saltiel AR. Phenotypic switching of adipose tissue macrophages with obesity is generated by spatiotemporal differences in macrophage subtypes. Diabetes 57, 3239-3246, 2008.

Miyazaki Y, Glass L, Triplitt C, Wajcberg E, Mandarino LJ, DeFronzo RA. Abdominal fat distribution and peripheral and hepatic insulin resistance in type 2 diabetes mellitus. Am J Physiol Endocrinol Metab 283, 1135-1143, 2002.

Nicklas BJ, Penninx BW, Cesari M, Kritchevsky SB, Newman AB, Kanaya AM, Pahor M, Jingzhong D, Harris TB. Association of visceral adipose tissue with incident myocardial infarction in older men and women: the health, aging and body composition study, Am J Epidemiol 160, 741-749, 2004.

Nguyen KD, Qiu Y, Cui X, Goh YP, Mwangi J, David T, Mukundan L, Brombacher F, Locksley RM, Chawla A. Alternatively activated macrophages produce catecholamines to sustain adaptive thermogenesis. Nature 480, 104-108, 2011.

Olefsky JM, Glass CK. Macrophages, inflammation and insulin resistance. Annu Rev Physiol 72, 219-246, 2010.

Qiu Y, Nguyen KD, Odegaard JI, Cui X, Tian X, Locksley RM, Palmiter RD, Chawla A. Eosinophils and type 2 cytokine signaling in macrophages orchestrate development of functional beige fat. Cell 157, 1292-1308, 2014.

Sam S, Haffner S, Davidson MH, D’Agostino RB Sr, Feinstein S, Kondos G, Perez A, Mazzone T. Relation of abdominal fat depots to systemic markers of inflammation in type 2 diabetes. Diabetes Care, 32, 932-937, 2009.

Seale P, Bjork B, Yang W, Kajimura S, Chin S, Kuang S, Scime A, Devarakonda S, Conroe HM, Erdjument-Bromage H, Tempst P, Rudnicki MA, Beier DR, Spiegelman BM. PRDM16 controls a brown fat/skeletal muscle switch. Nature 454, 961-967, 2008. 
Vargovic P, Ukropec J, Laukova M, Cleary S, Manz B, Pacak K, Kvetnansky R. Adipocytes as a new source of catecholamine production. FEBS Lett 585, 2279-2284, 2011.

Vargovic P, Ukropec J, Laukova M, Kurdiova T, Balaz M, Manz B, Ukropcova B, Kvetnansky R. Repeated immobilization stress induces catecholamine production in rat mesenteric adipocytes. Stress 16, 340-352, 2013.

Vargovic P, Laukova M, Ukropec J, Manz G, Kvetnansky R. Lipopolysaccharide induces catecholamine production in mesenteric adipose tissue of rats previously exposed to immobilization stress. Stress 2016. [Epub ahead of print].

Weisberg SP, McCann D, Desai M, Rosenbaum M, Leibel RL, Ferrante AW. Obesity is associated with macrophage accumulation in adipose tissue. J Clin Invest 112, 1796-1808, 2003.

Wu J, Bostrom P, Sparks LM, Ye L, Choi JH, Giang AH, Khandekar M, Virtanen KA, Nuutila P, Schaart G, Huang K, Tu H, van Marken Lichtenbelt WD, Hoeks J, Enerbäck S, Schrauwen P, Spiegelman BM. Beige adipocytes are a distinct type of thermogenic fat cell in mouse and human. Cell 150, 366-376, 2012.

Yang YK, Chen M, Clements RH, Abrams GA, Aprahamian CJ, Harmon CM. Human mesenteric adipose tissue plays unique role versus subcutaneous and omental fat in obesity related diabetes. Cell Physiol Biochem 22, $531-538,2008$

Young P, Wilson S, Arch JR. Prolonged beta-adrenoceptor stimulation increases the amount of GDP-binding protein in brown adipose tissue mitochondria. Life Sci 34, 1111-1117, 1984. 